\title{
Risk Factors for Mortality in Patients with COVID-19 in New York City
}

\author{
Takahisa Mikami, M.D. ${ }^{1,2}$, Hirotaka Miyashita, M.D. ${ }^{1,2}$, Takayuki Yamada, M.D. ${ }^{1,2}$, \\ Matthew Harrington, M.D. ${ }^{1,2}$, Daniel Steinberg, M.D. ${ }^{1,2}$, Andrew Dunn, M.D. ${ }^{1,3}$, and \\ Evan Siau, M.D. ${ }^{7,2}$
}

'Department of Medicine, Icahn School of Medicine at Mount SinaiNew York, NY, USA; ${ }^{2}$ Department of Medicine, Mount Sinai Beth Israel 281 First Ave, Box \#218, New York, NY, USA; ${ }^{3}$ Department of Medicine, Mount Sinai HospitalNew York, NY, USA.

BACKGROUND: New York City emerged as an epicenter of the coronavirus disease 2019 (COVID-19) pandemic. OBJECTIVE: To describe the clinical characteristics and risk factors associated with mortality in a large patient population in the USA.

DESIGN: Retrospective cohort study.

PARTICIPANTS: 6493 patients who had laboratoryconfirmed COVID-19 with clinical outcomes between March 13 and April 17, 2020, who were seen in one of the 8 hospitals and/or over 400 ambulatory practices in the New York City metropolitan area

MAIN MEASURES: Clinical characteristics and risk factors associated with in-hospital mortality.

KEY RESULTS: A total of 858 of 6493 (13.2\%) patients in our total cohort died: $52 / 2785$ (1.9\%) ambulatory patients and 806/3708 (21.7\%) hospitalized patients. Cox proportional hazard regression modeling showed an increased risk of in-hospital mortality associated with age older than 50 years (hazard ratio [HR] 2.34, CI 1.47-3.71), systolic blood pressure less than $90 \mathrm{mmHg}$ (HR 1.38, CI 1.06-1.80), a respiratory rate greater than 24 per min (HR 1.43, CI 1.13-1.83), peripheral oxygen saturation less than 92\% (HR 2.12, CI 1.56-2.88), estimated glomerular filtration rate less than $60 \mathrm{~mL} / \mathrm{min} / 1.73 \mathrm{~m}^{2}$ (HR $1.80, \mathrm{CI}$ 1.60-2.02), IL-6 greater than $100 \mathrm{pg} / \mathrm{mL}$ (HR 1.50, CI 1.12-2.03), D-dimer greater than $2 \mathrm{mcg} / \mathrm{mL}$ (HR 1.19, CI 1.02-1.39), and troponin greater than $0.03 \mathrm{ng} / \mathrm{mL}$ (HR 1.40, CI 1.23-1.62). Decreased risk of in-hospital mortality was associated with female sex (HR 0.84, CI 0.77-0.90), African American race (HR 0.78 CI 0.650.95), and hydroxychloroquine use (HR 0.53, CI 0.410.67).

CONCLUSIONS: Among patients with COVID-19, older age, male sex, hypotension, tachypnea, hypoxia, impaired renal function, elevated D-dimer, and elevated troponin were associated with increased in-hospital mortality and hydroxychloroquine use was associated with decreased in-hospital mortality.

Electronic supplementary material The online version of this article (https://doi.org/10.1007/s11606-020-05983-z) contains supplementary material, which is available to authorized users.

Received May 6, 2020

Accepted June 11, 2020

Published online June 30, 2020
J Gen Intern Med 36(1):17-26

DOI: $10.1007 / \mathrm{s} 11606-020-05983-Z$

(C) Society of General Internal Medicine 2020

\section{INTRODUCTION}

The coronavirus disease 2019 (COVID-19) is a pandemic that has impacted medical systems, societies, and economies worldwide. The first case of COVID-19, caused by severe acute respiratory syndrome 2 virus (SARS-CoV-2) ${ }^{1}$, was reported in China in December 2019². The virus has spread globally at a rapid pace, resulting in more than 2 million confirmed cases as of April 17, 20203. In recent weeks, New York City has emerged as an epicenter of the pandemic, with over 120,000 confirmed cases and over 13,000 deaths due to confirmed or probable COVID-19 death as of April 17, $2020^{4}$. Studies of the clinical characteristics and epidemiologic characteristics of COVID-19 have been conducted in countries experiencing outbreaks earlier than the USA ${ }^{5-11}$. Large-scale observational data of the clinical characteristics and outcomes of COVID-19 in the population of the USA are scarce. In this study, we describe the clinical characteristics of COVID-19 in ambulatory and inpatient settings and identify risk factors associated with mortality in hospitalized patients.

\section{METHODS}

\section{Study Design and Participants}

A multicenter retrospective cohort study of patients with COVID-19 patients was conducted using the medical records of the Mount Sinai Health System, a large urban health system of 8 hospitals and more than four hundred ambulatory practices in the New York City metropolitan area. Patients with a positive SARS-CoV-2 test result and an encounter with a healthcare provider for COVID-19 between March 12 and April 17, 2020, were included in this study. A confirmed case of COVID-19 was defined as a positive result on reversetranscriptase-polymerase-chain-reaction (RT-PCR) assay of nasopharyngeal swab specimens. The study population was dichotomized into ambulatory and hospitalized groups. The former included patients whose encounter was an office visit, 
emergency department (ED) visit, or telehealth/telemedicine. Inpatients and ambulatory patients who were subsequently admitted to the hospital were included in the hospitalized group.

Both groups were further subdivided into survivors and non-survivors. Ambulatory non-survivors were patients who had expired prior to presentation to the ED, who had expired in the ED prior to admission to the hospital units, or who had an office or telemedicine encounter and were later found out to be deceased. Ambulatory survivors included all other ambulatory patients. Hospitalized non-survivors were patients who had expired as of April 17, 2020. Hospitalized survivors were patients who had been discharged home or to other facilities as of April 17, 2020.

Icahn School of Medicine at Mount Sinai has waived informed consent and Institutional Review Board approval because the study used a de-identified database.

\section{Definitions}

The following covariates were extracted from the database: patients' age, sex, ethnicity, race, smoking status, vital signs including temperature, peripheral oxygen saturation $\left(\mathrm{SpO}_{2}\right)$, heart rate, respiratory rate (RR), blood pressure (BP), body mass index (BMI), and laboratory results including white blood cell count (WBC), D-dimer, interleukin-6 (IL-6), hemoglobin, estimated glomerular filtration rate (eGFR), alanine aminotransferase (ALT), aspartate aminotransferase (AST), C-reactive protein (CRP), procalcitonin, ferritin, lactate dehydrogenase (LDH), fibrinogen (FBG), interleukin-6 (IL-6), comorbidities, and treatments.

\section{Statistical Analysis}

Continuous variables were reported as median with interquartile range. Categorical variables were expressed as proportions. Temporary changes of vital signs and laboratory values in survivors and non-survivors for the first 14 days after admission were assessed. To illustrate the risk associated with changes in the continuous variables, including vital signs and laboratory values, multivariate generalized additive models were used to calculate the odds ratio (OR) for mortality, with each median value set as a reference (i.e., $\mathrm{OR}=1$ ). The hazard ratio (HR) of each variable for mortality risk was assessed using univariate Cox proportional hazard regression model. To account for missing data values for laboratory results, we introduced multiple imputation, which is a procedure used to replace missing values with other plausible values by creating multiple filling-in patterns to avert bias caused by missing data. Using the dataset with imputed values, univariate and multivariate Cox model were fit to calculate HR.

The multivariate Cox model was adjusted for the following variables assessed in the univariate Cox model: patients' age, sex, race, cigarette use history, past medical history of asthma, hypertension, diabetes, or cancer, systolic $\mathrm{BP}, \mathrm{RR}, \mathrm{SpO}_{2}$, $\mathrm{BMI}$, initial laboratory values (lymphocyte proportion, D- dimer, IL-6), and hydroxychloroquine use. For this Cox regression analysis, we excluded variables from the univariable analysis if their between-group differences were not significant, if the number of events was too small to calculate hazard ratios, or if they had collinearity with other significant values. Each hospital was considered by the clustering term in the Cox proportional hazard model analysis where the clustering effect associated with hospitals was accounted for by the robust sandwich estimator. Preliminary confirmation of predictability of the Cox proportional hazard model demonstrated the area under the curve (AUC) to be 0.808 (95\% CI, 0.790-0.825, Supplementary Figure 1). To investigate the effect of hydroxychloroquine while addressing the imbalance among treatment groups, we introduced inverse probability weighting (IPTW) based on propensity scoring to control for observed differences in baseline characteristics between treatment group and control group. IPTW was calculated based on the same variables as used in the Cox regression models, except for hydroxychloroquine use. We then fitted an IPTW-adjusted Cox with doubly robust methods. Survival curves with stratification for hydroxychloroquine were constructed using the Kaplan-Meier method. All statistical analyses were performed using version 3.6.2 of the $\mathrm{R}$ programming language ( $\mathrm{R}$ Project for Statistical Computing; R Foundation).

\section{RESULTS}

\section{Demographic and Clinical Characteristics}

Between March 13 and April 17, there were 6493 confirmed COVID-19 cases, including 2785 (42.9\%) ambulatory patients and 3708 (57.1\%) hospitalized patients. The demographics, clinical characteristics, and laboratory findings are shown in Table 1. The median age of the group was 59 (interquartile range [IQR] 43 to 72 ) with $66.6 \%$ of the patients older than 50 years of age. $45.5 \%$ of the patients were female. Based on patients' self-reported race, $26.9 \%$ were white, $24.1 \%$ were African American, $4.4 \%$ were Asian, and $44.7 \%$ were other. Based on self-reported ethnicity, $57.5 \%$ were NonHispanic, 25.4\% were Hispanic, and the rest were unknown or not reported.

\section{Ambulatory and Hospitalized Comparison}

The median age was 47 years old in the ambulatory group (IQR 34 to 60) and 66 years old in the hospitalized group (IQR 55 to 78 ). 858 patients died (13.2\%): 52 patients in the ambulatory group (1.9\%) and 806 patients in the hospitalized group (21.7\%). Among ambulatory patients, $69 \%$ were emergency room encounters without hospital admission, $18.2 \%$ were office-based encounters, and $1.4 \%$ were telemedicine encounters.

Compared with that of ambulatory patients, a higher proportion of hospitalized patients were older, were male, or had a history of cigarette use. Hospitalized patients were more likely 
Table 1 Clinical Characteristics of the Patients with COVID-19

\begin{tabular}{|c|c|c|c|}
\hline & Total $(n=6493)$ & Ambulatory $(n=2785)$ & Hospitalized $(n=3708)$ \\
\hline \multicolumn{4}{|l|}{ Demographics } \\
\hline Age (median [IQR]) & $59[43,72]$ & $47[34,60]$ & $66[55,78]$ \\
\hline \multicolumn{4}{|l|}{ Age-no./total no. (\%) } \\
\hline$<50$ years old & $2169 / 6493(33.4)$ & $1531 / 2785(55.0)$ & $638 / 3708(17.2)$ \\
\hline $50-74$ years old & $2996 / 6493(46.1)$ & $1081 / 2785(38.8)$ & $1915 / 3708(51.6)$ \\
\hline$\geq 75$ years old & $1328 / 6493(20.5)$ & $173 / 2785(6.2)$ & $1155 / 3708(31.1)$ \\
\hline Female-no./total no. (\%) & $2955 / 6493(45.5)$ & $1362 / 2785(48.9)$ & $1593 / 3708(43.0)$ \\
\hline \multicolumn{4}{|l|}{ Race - no./total no. $(\%)$} \\
\hline White & $1745 / 6493(26.9)$ & $817 / 2785(29.3)$ & $928 / 3708(25.0)$ \\
\hline African American & $1564 / 6493(24.1)$ & $650 / 2785(23.3)$ & $914 / 3708(24.6)$ \\
\hline Asian & $283 / 6493(4.4)$ & $127 / 2785(4.6)$ & $156 / 3708(4.2)$ \\
\hline Others & $2901 / 6493(44.7)$ & $1191 / 2785(42.8)$ & $1710 / 3708(46.1)$ \\
\hline \multicolumn{4}{|l|}{ Ethnicity — no./total no. (\%) } \\
\hline Non-Hispanic & $3734 / 6493(57.5)$ & $1543 / 2785(55.4)$ & $2191 / 3708(59.1)$ \\
\hline Hispanic & $1652 / 6493(25.4)$ & $635 / 2785(22.8)$ & $1017 / 3708(27.4)$ \\
\hline Unknown & $1107 / 6493(17.0)$ & $607 / 2785(21.8)$ & $500 / 3708(13.5)$ \\
\hline History of cigarette use - no./total no. (\%) & $1338 / 6493$ (20.6) & $429 / 2785(15.4)$ & $909 / 3708(24.5)$ \\
\hline Body mass index $\left(\mathrm{kg} / \mathrm{m}^{2}\right)$ (median [IQR]) & $27.7[24.3,32.4]$ & $27.4[24.1,31.9]$ & $27.9[24.3,32.6]$ \\
\hline Body mass index $\geq 30 \mathrm{~kg} / \mathrm{m}^{2}-$ no./total no. (\%) & $1557 / 4399(35.4)$ & $359 / 1119(32.1)$ & $1198 / 3280(36.5)$ \\
\hline \multicolumn{4}{|l|}{ Encounter type-no./total no. (\%) } \\
\hline Hospital & $5631 / 6493(86.7)$ & 1923/2785 (69.0) & $3708 / 3708(100.0)$ \\
\hline Clinic/office & $506 / 6493(7.8)$ & $506 / 2785(18.2)$ & $0 / 3708(0.0)$ \\
\hline Phone/telemedicine & $318 / 6493(4.9)$ & $318 / 2785(11.4)$ & $0 / 3708(0.0)$ \\
\hline Others & $38 / 6493(0.6)$ & $38 / 2785(1.4)$ & $0 / 3708(0.0)$ \\
\hline \multicolumn{4}{|l|}{ Past medical history-no./total no. (\%) } \\
\hline Asthma & $271 / 6493(4.2)$ & $98 / 2785(3.5)$ & $173 / 3708(4.7)$ \\
\hline Chronic pulmonary obstructive disease & $176 / 6493(2.7)$ & $26 / 2785(0.9)$ & $150 / 3708(4.0)$ \\
\hline Hypertension & $1637 / 6493(25.2)$ & $365 / 2785(13.1)$ & $1272 / 3708(34.3)$ \\
\hline Obesity & $418 / 6493(6.4)$ & $130 / 2785(4.7)$ & $288 / 3708(7.8)$ \\
\hline Diabetes & $1151 / 6493(17.7)$ & $250 / 2785(9.0)$ & $901 / 3708(24.3)$ \\
\hline Chronic kidney disease & $525 / 6493(8.1)$ & $94 / 2785(3.4)$ & 431/3708 (11.6) \\
\hline Human immunodeficiency virus infection & $98 / 6493(1.5)$ & $34 / 2785(1.2)$ & $64 / 3708(1.7)$ \\
\hline Cancer & $413 / 6493(6.4)$ & $159 / 2785(5.7)$ & $254 / 3708(6.9)$ \\
\hline \multicolumn{4}{|l|}{ Vital signs - no./total no. (\%) } \\
\hline Temperature $\geq 39^{\circ} \mathrm{C}$ & $1215 / 6039(20.1)$ & $113 / 2332(4.8)$ & $1102 / 3707(29.7)$ \\
\hline \multicolumn{4}{|l|}{ Peripheral oxygen saturation $\left(\mathrm{SpO}_{2}\right)$} \\
\hline$>92 \%$ & 4938/5702 (86.6) & 2140/2201 (97.2) & 2798/3501 (79.9) \\
\hline $88-92 \%$ & $502 / 5702(8.8)$ & $39 / 2201(1.8)$ & $463 / 3501(13.2)$ \\
\hline$\leq 87 \%$ & $262 / 5702(4.6)$ & $22 / 2201(1.0)$ & $240 / 3501(6.9)$ \\
\hline Heart rate $>120$ beats per min & $539 / 5973(9.0)$ & $116 / 2265(5.1)$ & 423/3708 (11.4) \\
\hline \multicolumn{4}{|l|}{ Respiratory rate } \\
\hline$\leq 24$ per $\min$ & $5155 / 5811(88.7)$ & $2050 / 2103(97.5)$ & $3105 / 3708(83.7)$ \\
\hline $25-30$ per min & $390 / 5811(6.7)$ & $36 / 2103(1.7)$ & $354 / 3708(9.5)$ \\
\hline$>30$ per $\min$ & $266 / 5811(4.6)$ & $17 / 2103(0.8)$ & $249 / 3708(6.7)$ \\
\hline Systolic blood pressure $<90 \mathrm{mmHg}$ & $121 / 5834(2.1)$ & $26 / 2138(1.2)$ & $95 / 3696(2.6)$ \\
\hline Diastolic blood pressure $<60 \mathrm{mmHg}$ & $664 / 5834$ (11.4) & $155 / 2138(7.2)$ & $509 / 3696(13.8)$ \\
\hline \multicolumn{4}{|l|}{ Laboratory results } \\
\hline WBC $\left(\times 10^{3} / \mu \mathrm{L}\right)($ median $[\mathrm{IQR}])$ & $7.30[5.40,10.30]$ & $6.20[4.66,8.36]$ & $7.60[5.50,10.6]$ \\
\hline$<4.0 \times 10^{3} / \mu \mathrm{L}-$ no./total no. $(\%)$ & $380 / 4353(8.7)$ & $106 / 703(15.1)$ & $274 / 3650(7.5)$ \\
\hline $4.0-8.0 \times 10^{3} / \mu \mathrm{L}-$ no./total no. $(\%)$ & $3246 / 4353(74.6)$ & $538 / 703(76.5)$ & $2708 / 3650(74.2)$ \\
\hline$>12.0 \times 10^{3} / \mu \mathrm{L}-$ no. $/$ total no. $(\%)$ & $727 / 4353(16.7)$ & $59 / 703(8.4)$ & $668 / 3650(18.3)$ \\
\hline Neutrophil & & & \\
\hline Count $\left(\times 10^{3} / \mu \mathrm{L}\right)($ median $[\mathrm{IQR}])$ & $5.60[3.80,8.20]$ & $4.20[2.90,6.12]$ & $5.80[4.00,8.50]$ \\
\hline Percentage (median [IQR]) & $78.3[70.0,85.0]$ & $72.0[63.2,79.8]$ & $79.4[71.8,85.8]$ \\
\hline Percentage $>78-$ no./total no. $(\%)$ & $1196 / 2345(51.0)$ & $116 / 388(29.9)$ & $1080 / 1957(55.2)$ \\
\hline Lymphocyte & & & \\
\hline Count $\left(\times 10^{3} / \mu \mathrm{L}\right)($ median $[\mathrm{IQR}])$ & $0.90[0.60,1.30]$ & $1.10[0.80,1.40]$ & $0.90[0.60,1.20]$ \\
\hline Percentage (median [IQR]) & $12.3[7.90,19.0]$ & $17.4[11.3,25.0]$ & $11.7[7.40,17.6]$ \\
\hline Percentage $\leq 12-$ no./total no. $(\%)$ & $1242 / 2345(53.0)$ & $131 / 388(33.8)$ & $1111 / 1957(56.8)$ \\
\hline Hemoglobin (g/dL) (median [IQR]) & $13.3[11.97,14.5]$ & $13.8[12.7,14.9]$ & $13.2[11.8,14.5]$ \\
\hline$<12 \mathrm{~g} / \mathrm{dL}-$ no./total no. $(\%)$ & $1626 / 2204(73.8)$ & $273 / 321(85.0)$ & $1353 / 1883(71.9)$ \\
\hline Platelet count $\left(\times 10^{3} / \mu \mathrm{L}\right)($ median $[\mathrm{IQR}])$ & $211.0[160.8,272.3]$ & $204.0[164.0,268.0]$ & $211.0[160.0,273.0]$ \\
\hline$>200 \times 10^{3} / \mu \mathrm{L}-$ no./total no. $(\%)$ & $1299 / 2344(55.4)$ & $208 / 385(54.0)$ & $1091 / 1959(55.7)$ \\
\hline eGFR $\left(\mathrm{mL} / \mathrm{min} / 1.73 \mathrm{~m}^{2}\right)($ median [IQR]) & $68.8[40.1,94.3]$ & $78.1[57.28,99.6]$ & $66.3[37.3,93.40]$ \\
\hline$>60 \mathrm{~mL} / \mathrm{min} / 1.73 \mathrm{~m}^{2}-$ no. $/$ total no. $(\%)$ & $2505 / 4295(58.3)$ & $490 / 687(71.3)$ & $2015 / 3608(55.8)$ \\
\hline $30-60 \mathrm{~mL} / \mathrm{min} / 1.73 \mathrm{~m}^{2}-$ no./total no. $(\%)$ & $1023 / 4295(23.8)$ & $139 / 687(20.2)$ & $884 / 3608(24.5)$ \\
\hline$<30 \mathrm{~mL} / \mathrm{min} / 1.73 \mathrm{~m}^{2}-$ no./total no. $(\%)$ & $767 / 4295(17.9)$ & $58 / 687(8.4)$ & $709 / 3608(19.7)$ \\
\hline Alanine aminotransferase $(\mathrm{U} / \mathrm{L})$ (median $[\mathrm{IQR}])$ & $30.0[19.0,51.0]$ & $29.0[19.0,46.0]$ & $30.0[19.0,52.0]$ \\
\hline$>40 \mathrm{U} / \mathrm{L}-$ no./total no. $(\%)$ & $1370 / 4009(34.2)$ & $146 / 490(29.8)$ & $1224 / 3519(34.8)$ \\
\hline Aspartate aminotransferase (U/L) (median [IQR]) & $43.0[29.0,69.0]$ & $35.0[24.0,54.0]$ & $44.0[29.0,71.0]$ \\
\hline$>40 \mathrm{U} / \mathrm{L}-$ no./total no. $(\%)$ & $2111 / 3952(53.4)$ & $187 / 458(40.8)$ & $1924 / 3494(55.1)$ \\
\hline C-reactive protein $(\mathrm{mg} / \mathrm{L})($ median $[\mathrm{IQR}])$ & $125.4[60.3,215.3]$ & $89.1[37.1,159.6]$ & $127.8[62.1,218.9]$ \\
\hline$>150 \mathrm{mg} / \mathrm{L}-$ no./total no. $(\%)$ & $623 / 1491(41.8)$ & $34 / 109(31.2)$ & $589 / 1382(42.6)$ \\
\hline Procalcitonin (ng/mL) (median [IQR]) & $0.20[0.08,0.65]$ & $0.10[0.05,0.31]$ & $0.21[0.08,0.68]$ \\
\hline
\end{tabular}


Table 1. (continued)

\begin{tabular}{|c|c|c|c|}
\hline & Total $(n=6493)$ & Ambulatory $(n=2785)$ & Hospitalized $(n=3708)$ \\
\hline$>0.5 \mathrm{ng} / \mathrm{mL}-$ no. $/$ total no. $(\%)$ & $3143 / 3143(100.0)$ & $212 / 212(100.0)$ & $2931 / 2931(100.0)$ \\
\hline Ferritin (ng/mL) (median [IQR]) & $748[339,1769]$ & $518.0[259,1347]$ & $759[351,1797]$ \\
\hline$>400 \mathrm{ng} / \mathrm{mL}-$ no./total no. (\%) & $2278 / 3234(70.4)$ & $128 / 221(57.9)$ & $2150 / 3013(71.4)$ \\
\hline Interleukin-6, serum $(\mathrm{pg} / \mathrm{mL})$ (median [IQR]) & $68.2[32.8,145.8]$ & $37.4[21.2,57.3]$ & $68.5[33.0,146.1]$ \\
\hline$>100 \mathrm{pg} / \mathrm{mL}-$ no./total no. $(\%)$ & $414 / 1150(36.0)$ & $3 / 13(23.1)$ & $411 / 1137(36.1)$ \\
\hline Lactate dehydrogenase (U/L) (median [IQR]) & $429.0[322.0,583.0]$ & $361.5[282.0,488.0]$ & $435.0[326.0,585.5]$ \\
\hline$>440 \mathrm{U} / \mathrm{L}-$ no./total no. $(\%)$ & $1495 / 3143(47.6)$ & $66 / 212(31.1)$ & $1429 / 2931(48.8)$ \\
\hline Fibrinogen (mg/dL) (median [IQR]) & $633.0[512.0,755.0]$ & $595.0[486.5,691.5]$ & $634.0[512.3,758.0]$ \\
\hline$>400 \mathrm{mg} / \mathrm{dL}-$ no./total no. $(\%)$ & $1540 / 1705(90.3)$ & $52 / 55(94.5)$ & $1488 / 1650(90.2)$ \\
\hline D-dimer $(\mu \mathrm{g} / \mathrm{mL})($ median [IQR]) & $1.53[0.85,3.01]$ & $1.12[0.61,2.29]$ & $1.56[0.88,3.04]$ \\
\hline$>2 \mu \mathrm{g} / \mathrm{mL}-$ no./total no. (\%) & $1172 / 2984(39.3)$ & $59 / 214(27.6)$ & $1113 / 2770(40.2)$ \\
\hline Troponin (ng/dL) (median [IQR]) & $0.03[0.02,0.10]$ & $0.02[0.01,0.05]$ & $0.03[0.02,0.10]$ \\
\hline$>0.03 \mathrm{ng} / \mathrm{dL}-$ no./total no. $(\%)$ & $1397 / 2805(49.8)$ & $85 / 279(30.5)$ & $1312 / 2526(51.9)$ \\
\hline \multicolumn{4}{|l|}{ Medications - no./total no. $(\%)$} \\
\hline Hydroxychloroquine & $2863 / 6493(44.1)$ & $50 / 2785(1.8)$ & 2813/3708 (75.9) \\
\hline Azithromycin & $2785 / 6493(42.9)$ & $193 / 2785(6.9)$ & $2592 / 3708(69.9)$ \\
\hline Death (median [IQR]) & $858 / 6493(13.2)$ & $52 / 2785(1.9)$ & $806 / 3708(21.7)$ \\
\hline
\end{tabular}

$I Q R$, interquartile range; WBC, white blood cell count; eGFR, estimated glomerular filtration rate

to have coexisting medical conditions including asthma, chronic obstructive pulmonary disease (COPD), hypertension, obesity, diabetes mellitus (DM), chronic kidney disease (CKD), and cancer. Hospitalized patients were more likely to have abnormal vital signs and abnormal laboratory values including higher WBC count, lymphocyte, and neutrophil counts, higher levels of AST, CRP, procalcitonin, ferritin, IL-6, LDH, D-dimer, and troponin, and lower levels of eGFR and hemoglobin. Clinical characteristics of hospitalized patients stratified by age group, gender, race, and hydroxychloroquine use are shown in Supplementary Tables 2, 3, 4, and 5 , respectively.

\section{Survivors and Non-Survivors}

Clinical characteristics of the 2014 survivors and 806 nonsurvivors in the hospitalized group are shown in Table 2 (Supplementary Table 1 for the ambulatory group). The median number of days to discharge for survivors was 5 days (IQR, 3 to 9 days). The median number of days to death for non-survivors was also 5 days (IQR, 3 to 9 days). Compared with survivors, non-survivors were older and the higher proportion were male. Non-survivors were more likely to have a history of cigarette use and coexisting medical conditions including COPD, hypertension, DM, and CKD.

Temporal changes of vital signs and laboratory values in survivors and non-survivors during hospitalization are shown in Figure 1. Throughout hospitalization, non-survivors had higher heart rate and respiratory rate and lower oxygen saturation compared with survivors. Initial laboratory findings of non-survivors demonstrated higher WBC count and higher levels of D-dimer, IL-6, AST, CRP, procalcitonin, ferritin, LDH, fibrinogen, and troponin. Throughout hospitalization, non-survivors had higher WBC count, neutrophil proportion, $\mathrm{LDH}$, and ferritin levels, and lower eGFR and lymphocyte proportion. Non-survivors also had higher levels of CRP, Ddimer, and IL-6 in the first week of hospitalization. Non- survivors showed a marked increase in LDH, CRP, D-dimer, AST, ALT, and procalcitonin on day 1 after admission. Both groups had a trend of decreasing hemoglobin levels and increasing platelet counts during hospitalization; however, a more pronounced decrease in hemoglobin levels was seen in non-survivors, while an increase in platelet counts was greater for survivors. The generalized additive models demonstrated correlations between laboratory values and increased odds of in-hospital mortality which are similar to the difference observed between hospitalized survivors and non-survivors (Supplementary Figure 3).

\section{Treatment}

The majority of hospitalized patients received hydroxychloroquine (74.6\% of survivors and $71.3 \%$ of non-survivors) and azithromycin $(67.4 \%$ of survivors and $71.3 \%$ of non-survivors). Fewer hospitalized patients received other medications such as remdesivir, anakinra, tocilizumab, or sarilumab (Table 2). The majority of ambulatory patients did not receive hydroxychloroquine or azithromycin. Kaplan-Meier estimate showed lower mortality in hospitalized patients who received hydroxychloroquine $(\log$ rank $P$ value $<0.001)$ (Supplementary Figure 4).

\section{Risk Factors Associated with Mortality in Hospitalized Patients}

The results of multivariate Cox proportional hazard regression models are shown in Table 3 (univariate models are shown in Supplementary Table 6). Of 3708 hospitalized patients, 888 patients remained hospitalized as of April 7 and were not included in the analysis. In the multivariate analysis, factors associated with a higher risk of in-hospital mortality included age over 50, systolic blood pressure less than $90 \mathrm{mmHg}$, a respiratory rate greater than 24 per min, $\mathrm{SpO}_{2}$ less than $92 \%$, eGFR less than $60 \mathrm{~mL} / \mathrm{min} / 1.73 \mathrm{~m}^{2}$, IL-6 greater than $100 \mathrm{pg} /$ 
Table 2 Clinical Characteristics of the Hospitalized Patients with COVID-19

\begin{tabular}{|c|c|c|c|}
\hline & Survivors $(n=2014)$ & Non-survivors $(n=806)$ & In-hospital $(n=888)$ \\
\hline \multicolumn{4}{|l|}{ Demographics } \\
\hline Age (median [IQR]) & $62[49,73]$ & $76[65,85]$ & $68[58,78]$ \\
\hline \multicolumn{4}{|l|}{ Age-no./total no. (\%) } \\
\hline Age $<50$ years old & $505 / 2014(25.1)$ & $30 / 806(3.7)$ & 103/888 (11.6) \\
\hline Age: $60-79$ years old & $1086 / 2014(53.9)$ & $343 / 806(42.6)$ & $486 / 888(54.7)$ \\
\hline Age $\geq 75$ years old & $423 / 2014(21.0)$ & $433 / 806(53.7)$ & $299 / 888(33.7)$ \\
\hline Female-no./total no. (\%) & $886 / 2014(44.0)$ & $323 / 806(40.1)$ & $384 / 888(43.2)$ \\
\hline \multicolumn{4}{|l|}{ Race-no./total no. (\%) } \\
\hline White & $496 / 2014(24.6)$ & 243/806 (30.1) & $189 / 888(21.3)$ \\
\hline African American & $502 / 2014(24.9)$ & $194 / 806(24.1)$ & $218 / 888(24.5)$ \\
\hline Asian & $81 / 2014(4.0)$ & $36 / 806(4.5)$ & $39 / 888(4.4)$ \\
\hline Others & $935 / 2014(46.4)$ & $333 / 806(41.3)$ & $442 / 888(49.8)$ \\
\hline \multicolumn{4}{|l|}{ Ethnicity-no./total no. (\%) } \\
\hline Non-Hispanic & $1180 / 2014(58.6)$ & $502 / 806(62.3)$ & $509 / 888(57.3)$ \\
\hline Hispanic & $594 / 2014(29.5)$ & $171 / 806(21.2)$ & $252 / 888(28.4)$ \\
\hline Unknown & $240 / 2014(11.9)$ & $133 / 806(16.5)$ & $127 / 888(14.3)$ \\
\hline History of cigarette use - no./total no. (\%) & $455 / 2014(22.6)$ & $229 / 806(28.4)$ & $225 / 888(25.3)$ \\
\hline Body mass index $\left(\mathrm{kg} / \mathrm{m}^{2}\right)$ (median $\left.[\mathrm{IQR}]\right)$ & $28.07[24.6,32.6]$ & $27.6[23.9,32.5]$ & $27.5[24.0,32.61]$ \\
\hline Body mass index $\geq 30 \mathrm{~kg} / \mathrm{m}^{2}-$ no./total no. (\%) & $678 / 1828(37.1)$ & $237 / 662(35.8)$ & $283 / 790(35.8)$ \\
\hline \multicolumn{4}{|l|}{ Past medical history-no./total no. (\%) } \\
\hline Asthma & $97 / 2014(4.8)$ & $31 / 806(3.8)$ & $45 / 888(5.1)$ \\
\hline Chronic pulmonary obstructive disease & $60 / 2014(3.0)$ & $46 / 806(5.7)$ & $44 / 888(5.0)$ \\
\hline Hypertension & $606 / 2014(30.1)$ & $324 / 806(40.2)$ & $342 / 888(38.5)$ \\
\hline Obesity & $164 / 2014(8.1)$ & $57 / 806(7.1)$ & $67 / 888(7.5)$ \\
\hline Diabetes & $436 / 2014(21.6)$ & $221 / 806(27.4)$ & $244 / 888(27.5)$ \\
\hline Chronic kidney disease & $186 / 2014(9.2)$ & $131 / 806(16.3)$ & $114 / 888(12.8)$ \\
\hline Human immunodeficiency virus infection & $38 / 2014$ (1.9) & $11 / 806(1.4)$ & $15 / 888(1.7)$ \\
\hline Cancer & $125 / 2014(6.2)$ & $69 / 806(8.6)$ & $60 / 888(6.8)$ \\
\hline \multicolumn{4}{|l|}{ Vital signs-no./total no. $(\%)$} \\
\hline Temperature $\geq 39^{\circ} \mathrm{C}$ & $536 / 2014(26.6)$ & $294 / 805(36.5)$ & $272 / 888(30.6)$ \\
\hline \multicolumn{4}{|l|}{ Peripheral oxygen saturation $\left(\mathrm{SpO}_{2}\right)$} \\
\hline$>92 \%$ & $1700 / 1978(85.9)$ & $518 / 705(73.5)$ & $580 / 818(70.9)$ \\
\hline $88-92 \%$ & $204 / 1978(10.3)$ & $121 / 705(17.2)$ & $138 / 818(16.9)$ \\
\hline$\leq 87 \%$ & $74 / 1978(3.7)$ & $66 / 705(9.4)$ & $100 / 818(12.2)$ \\
\hline Heart rate $>120$ beats per min & $205 / 2014(10.2)$ & $97 / 806(12.0)$ & $121 / 888(13.6)$ \\
\hline \multicolumn{4}{|l|}{ Respiratory rate } \\
\hline$\leq 24$ per $\min$ & $1847 / 2014(91.7)$ & $605 / 806(75.1)$ & $653 / 888(73.5)$ \\
\hline $25-30$ per $\min$ & $105 / 2014(5.2)$ & $104 / 806(12.9)$ & $145 / 888(16.3)$ \\
\hline$>30$ per $\min$ & $62 / 2014(3.1)$ & $97 / 806(12.0)$ & $90 / 888(10.1)$ \\
\hline Systolic blood pressure $<90 \mathrm{mmHg}$ & $33 / 2008(1.6)$ & $36 / 804(4.5)$ & $26 / 884(2.9)$ \\
\hline Diastolic blood pressure $<60 \mathrm{mmHg}$ & $227 / 2008(11.3)$ & $158 / 804(19.7)$ & $124 / 884(14.0)$ \\
\hline \multicolumn{4}{|l|}{ Laboratory results } \\
\hline WBC $\left(\times 10^{3} / \mu \mathrm{L}\right)($ median $[\mathrm{IQR}])$ & $7.00[5.30,9.41]$ & $8.80[6.19,12.2]$ & $8.30[5.80,11.9]$ \\
\hline$<4.0 \times 10^{3} / \mu \mathrm{L}-$ no./total no. (\%) & $172 / 1967(8.7)$ & $47 / 796(5.9)$ & $55 / 887(6.2)$ \\
\hline $4.0-8.0 \times 10^{3} / \mu \mathrm{L}-$ no./total no. $(\%)$ & $1545 / 1967(78.5)$ & $542 / 796(68.1)$ & $621 / 887(70.0)$ \\
\hline$>12.0 \times 10^{3} / \mu \mathrm{L}-$ no. $/$ total no. $(\%)$ & $250 / 1967(12.7)$ & $207 / 796(26.0)$ & $211 / 887(23.8)$ \\
\hline \multicolumn{4}{|l|}{ Neutrophil } \\
\hline Count $\left(\times 10^{3} / \mu \mathrm{L}\right)($ median $[\mathrm{IQR}])$ & $5.20[3.60,7.50]$ & $7.10[4.90,10.5]$ & $6.30[4.20,9.30]$ \\
\hline Percentage (median [IQR]) & $77.7[70.0,84.1]$ & $81.8[74.0,87.7]$ & $82.0[75.2,87.1]$ \\
\hline Percentage $>78-$ no./total no. $(\%)$ & $533 / 1103(48.3)$ & $249 / 393(63.4)$ & $298 / 461(64.6)$ \\
\hline Lymphocyte & & & \\
\hline Count $\left(\times 10^{3} / \mu \mathrm{L}\right)($ median $[\mathrm{IQR}])$ & $0.90[0.70,1.30]$ & $0.80[0.50,1.10]$ & $0.80[0.50,1.10]$ \\
\hline Percentage (median [IQR]) & $13.0[8.60,19.2]$ & $9.20[5.70,15.0]$ & $10.1[6.30,15.5]$ \\
\hline Percentage $\leq 12-$ no./total no. $(\%)$ & $542 / 1103(49.1)$ & $266 / 393(67.7)$ & $303 / 461(65.7)$ \\
\hline Hemoglobin (g/dL) (median [IQR]) & $13.4[12.2,14.5]$ & $12.9[11.1,14.4]$ & $13.4[11.6,14.4]$ \\
\hline$<12 \mathrm{~g} / \mathrm{dL}-$ no./total no. $(\%)$ & $708 / 903(78.4)$ & $313 / 506(61.9)$ & $332 / 474(70.0)$ \\
\hline Platelet count $\left(\times 10^{3} / \mu \mathrm{L}\right)($ median $[\mathrm{IQR}])$ & $212.0[166.0,267.0]$ & $197.0[146.0,252.0]$ & $225.0[164.0,296.0]$ \\
\hline$>200 \times 10^{3} / \mu \mathrm{L}-$ no. $/$ total no. $(\%)$ & $629 / 1105(56.9)$ & $184 / 393(46.8)$ & $278 / 461(60.3)$ \\
\hline eGFR $\left(\mathrm{mL} / \mathrm{min} / 1.73 \mathrm{~m}^{2}\right)($ median $[\mathrm{IQR}])$ & $76.8[49.5,102.0]$ & $45.8[24.3,70.7]$ & $61.4[32.5,89.6]$ \\
\hline$>60 \mathrm{~mL} / \mathrm{min} / 1.73 \mathrm{~m}^{2}-$ no./total no. $(\%)$ & $1289 / 1925(67.0)$ & $264 / 796(33.2)$ & $462 / 887(52.1)$ \\
\hline $30-60 \mathrm{~mL} / \mathrm{min} / 1.73 \mathrm{~m}^{2}-$ no./total no. $(\%)$ & $383 / 1925(19.9)$ & $282 / 796(35.4)$ & $219 / 887(24.7)$ \\
\hline$<30 \mathrm{~mL} / \mathrm{min} / 1.73 \mathrm{~m}^{2}-$ no./total no. $(\%)$ & $253 / 1925(13.1)$ & $250 / 796(31.4)$ & $206 / 887(23.2)$ \\
\hline Alanine aminotransferase (U/L) (median [IQR]) & $29.0[18.0,52.0]$ & $32.0[20.0,54.0]$ & $29.0[18.0,53.0]$ \\
\hline$>40 \mathrm{U} / \mathrm{L}-$ no./total no. $(\%)$ & $634 / 1869(33.9)$ & $288 / 773(37.3)$ & $302 / 877(34.4)$ \\
\hline Aspartate aminotransferase (U/L) (median [IQR]) & $40.0[28.0,62.0]$ & $56.0[35.0,90.0]$ & $46.0[31.0,73.0]$ \\
\hline$>40 \mathrm{U} / \mathrm{L}-$ no./total no. $(\%)$ & $891 / 1856(48.0)$ & $522 / 762(68.5)$ & $511 / 876(58.3)$ \\
\hline C-reactive protein $(\mathrm{mg} / \mathrm{L})($ median $[\mathrm{IQR}])$ & $93.9[44.6,172.7]$ & $174.4[95.3,254.6]$ & $154.04[82.7,239.5]$ \\
\hline$>150 \mathrm{mg} / \mathrm{L}-$ no./total no. $(\%)$ & $203 / 657(30.9)$ & $178 / 315(56.5)$ & $208 / 410(50.7)$ \\
\hline Procalcitonin (ng/mL) (median [IQR]) & $0.13[0.06,0.37]$ & $0.47[0.18,1.53]$ & $0.27[0.11,0.82]$ \\
\hline$>0.5 \mathrm{ng} / \mathrm{mL}-$ no. $/$ total no. $(\%)$ & $1545 / 1545(100.0)$ & $581 / 581(100.0)$ & $805 / 805(100.0)$ \\
\hline Ferritin (ng/mL) (median [IQR]) & $637.5[287.8,1479.0]$ & $938.0[432.0,2186.0]$ & $928.0[434.0,2051.5]$ \\
\hline$>400 \mathrm{ng} / \mathrm{mL}-$ no./total no. $(\%)$ & $1017 / 1556(65.4)$ & $490 / 625(78.4)$ & $643 / 832(77.3)$ \\
\hline Interleukin-6, serum (pg/mL) (median [IQR]) & $45.8[23.3,82.4]$ & $152.4[79.1,303.8]$ & $78.4[40.7,152.1]$ \\
\hline$>100 \mathrm{pg} / \mathrm{mL}-$ no./total no. $(\%)$ & $117 / 582(20.1)$ & $187 / 287(65.2)$ & $107 / 268(39.9)$ \\
\hline
\end{tabular}


Table 2. (continued)

\begin{tabular}{|c|c|c|c|}
\hline & Survivors $(n=2014)$ & Non-survivors $(n=806)$ & In-hospital $(n=888)$ \\
\hline Lactate dehydrogenase $(\mathrm{U} / \mathrm{L})$ (median $[\mathrm{IQR}])$ & $391.0[303.0,500.0]$ & $511.0[382.0,758.0]$ & $478.0[364.0,650.0]$ \\
\hline$>440 \mathrm{U} / \mathrm{L}-$ no./total no. $(\%)$ & $586 / 1545(37.9)$ & $372 / 581(64.0)$ & $471 / 805(58.5)$ \\
\hline Fibrinogen (mg/dL) (median [IQR]) & $616.0[506.0,727.0]$ & $634.0[506.0,777.0]$ & $664.0[521.8,790.0]$ \\
\hline$>400 \mathrm{mg} / \mathrm{dL}-$ no./total no. $(\%)$ & 696/761 (91.5) & $302 / 349(86.5)$ & $490 / 540(90.7)$ \\
\hline D-dimer $(\mu \mathrm{g} / \mathrm{mL})($ median $[\mathrm{IQR}])$ & $1.25[0.73,2.35]$ & $2.29[1.29,4.00]$ & $1.79[1.05,3.51]$ \\
\hline$>2 \mu \mathrm{g} / \mathrm{mL}$ - no./total no. (\%) & $426 / 1407(30.3)$ & $315 / 548(57.5)$ & $372 / 815(45.6)$ \\
\hline Troponin (ng/dL) (median [IQR]) & $0.02[0.01,0.06]$ & $0.07[0.03,0.21]$ & $0.04[0.02,0.12]$ \\
\hline$>0.03 \mathrm{ng} / \mathrm{dL}-$ no./total no. $(\%)$ & $410 / 1108(37.0)$ & $504 / 718(70.2)$ & $398 / 700(56.9)$ \\
\hline Interleukin-1b (pg/mL) (median [IQR]) & $0.50[0.30,0.80]$ & $0.60[0.40,1.20]$ & $0.50[0.40,0.80]$ \\
\hline$>5$ pg/mL—no./total no. (\%) & $6 / 354(1.7)$ & $0 / 100(0.0)$ & $2 / 168(1.2)$ \\
\hline Interleukin-8 (pg/mL) (median [IQR]) & $34.6[22.2,54.6]$ & $60.2[37.9,112.5]$ & $46.2[32.0,75.2]$ \\
\hline$>5 \mathrm{pg} / \mathrm{mL}-$ no./total no. $(\%)$ & $423 / 424(99.8)$ & $115 / 115(100.0)$ & $197 / 198(99.5)$ \\
\hline Tumor necrosis factor alpha $(\mathrm{pg} / \mathrm{mL})$ (median $[\mathrm{IQR}])$ & $20.60[15.9,28.3]$ & $25.9[19.7,38.3]$ & $23.9[16.7,36.7]$ \\
\hline \multirow{2}{*}{\multicolumn{4}{|c|}{ Medications }} \\
\hline & & & \\
\hline \multicolumn{4}{|l|}{ Treatments } \\
\hline Hydroxychloroquine - no./total no. (\%) & $1502 / 2014(74.6)$ & $575 / 806(71.3)$ & 736/888 (82.9) \\
\hline Initiation (day) (median [IQR]) & $0.74[0.43,1.15]$ & $0.89[0.51,1.65]$ & $0.57[0.32,0.96]$ \\
\hline Azithromycin - no./total no. (\%) & $1357 / 2014(67.4)$ & $575 / 806(71.3)$ & $660 / 888(74.3)$ \\
\hline Initiation (day) (median [IQR]) & $0.11[0.00,0.45]$ & $0.11[0.01,0.49]$ & $0.10[-0.02,0.42]$ \\
\hline Remdesivir-no./total no. (\%) & $11 / 2014(0.5)$ & $2 / 806(0.2)$ & $43 / 888(4.8)$ \\
\hline Initiation (day) (median [IQR]) & $4.04[2.23,5.56]$ & $4.04[3.56,4.53]$ & $2.84[2.50,3.66]$ \\
\hline Anakinra- no./total no. (\%) & $0 / 2014(0.0)$ & $2 / 806(0.2)$ & $1 / 888(0.1)$ \\
\hline Initiation (day) (median [IQR]) & NA & $3.67[3.41,3.92]$ & $2.13[2.13,2.13]$ \\
\hline Tocilizumab-no./total no. (\%) & $47 / 2014(2.3)$ & $60 / 806(7.4)$ & $57 / 888(6.4)$ \\
\hline Initiation (day) (median [IQR]) & $2.25[1.37,4.26]$ & $3.16[2.05,5.83]$ & $3.61[1.95,6.02]$ \\
\hline Sarilumab-no./total no. $(\%)$ & $10 / 2014(0.5)$ & $12 / 806(1.5)$ & $10 / 888(1.1)$ \\
\hline Initiation (day) (median [IQR]) & $4.29[2.15,5.19]$ & $5.23[3.90,8.06]$ & $3.11[1.79,5.67]$ \\
\hline \multicolumn{4}{|l|}{ Anticoagulation } \\
\hline Heparin - no./total no. (\%) & $1233 / 2014(61.2)$ & $546 / 806(67.7)$ & $486 / 888(54.7)$ \\
\hline Enoxaparin -no./total no. (\%) & $766 / 2014(38.0)$ & $248 / 806(30.8)$ & $581 / 888(65.4)$ \\
\hline Apixaban-no./total no. (\%) & $189 / 2014(9.4)$ & $106 / 806(13.2)$ & $219 / 888(24.7)$ \\
\hline Rivaroxaban-no./total no. (\%) & $34 / 2014(1.7)$ & $17 / 806(2.1)$ & $13 / 888(1.5)$ \\
\hline Tissue plasminogen activator-no./total no. (\%) & $4 / 2014(0.2)$ & $40 / 806(5.0)$ & $36 / 888(4.1)$ \\
\hline Initiation of anticoagulation (day) (median [IQR]) & $0.40[0.20,0.74]$ & $0.43[0.24,0.81]$ & $0.33[0.15,0.67]$ \\
\hline \multicolumn{4}{|l|}{ Other variables } \\
\hline Length of stay (median [IQR]) & $5[3,9]$ & $5[3,10]$ & NA \\
\hline
\end{tabular}

$I Q R$, interquartile range; WBC, white blood cell count; eGFR, estimated glomerular filtration rate

$\mathrm{mL}$ (6.5 times upper limit of normal [ULN]), D-dimer greater than $2 \mathrm{mcg} / \mathrm{mL}$ ( 4 times ULN), and troponin greater than 0.03 $\mathrm{ng} / \mathrm{mL}$. Factors associated with a lower risk of in-hospital mortality included female sex, African American race, and hydroxychloroquine use. The adjustment with IPTW did not lead to a significant change in the HR of hydroxychloroquine (without IPTW: HR 0.53, CI 0.41-0.67; with IPTW: HR 0.53, CI 0.41-0.68).

\section{DISCUSSION}

We report a large retrospective cohort study of both ambulatory and hospitalized patients with COVID-19 from across the New York City metropolitan area. The clinical characteristics described here represent the first large retrospective cohort study from the US population in a city at the epicenter of the pandemic.

Early reports showed that COVID-19 had a mortality rate among all confirmed cases of $2 \%{ }^{12}$ which is significantly lower compared with that of $34 \%$ with MERS ${ }^{13}$ and $10 \%$ with $\mathrm{SARS}^{14}$. The mortality rate in hospitalized patients reported previously ranged from 4 to $28 \% \%^{2,7-9,11}$. The mortality rate of
$25.9 \%$ among hospitalized patients in our study may be explained by more severe disease in our total cohort, by a different reporting method, or by geographic variation.

We identified several risk factors associated with mortality in hospitalized patients with COVID-19 that have been previously reported including older age and male sex. We report additional risk factors associated with in-hospital mortality including low SBP, tachypnea, low $\mathrm{SpO}_{2}$, low eGFR, and higher levels of IL-6, D-dimer, and troponin levels.

The severity of coronavirus infection in humans has been previously described to increase during viral clearance suggesting pathogenicity arising from host immune response ${ }^{15}$. Our study confirmed again that older patients with COVID-19 hospitalization are at significantly higher risk of mortality. We did not observe any independent association between inhospital mortality and some of the common coexisting medical conditions including hypertension, diabetes, or cancer. However, using calculated GFR as a surrogate for CKD, we observed that decreased renal function was a risk factor for inhospital mortality, a finding that is consistent with previous studies $^{16}$.

IL-6 and other pro-inflammatory cytokines production are felt to be due to immune dysregulation rather than normal 
a
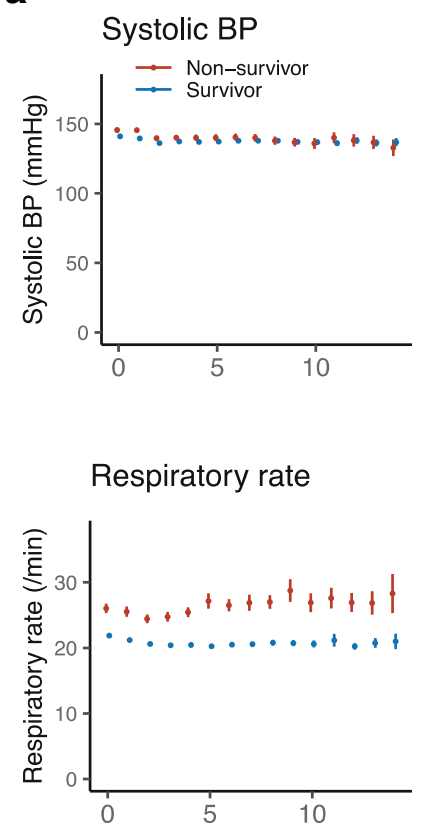

Diastolic BP

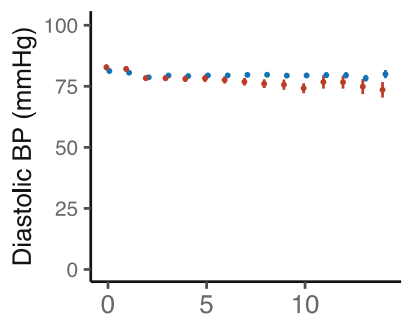

Temperature

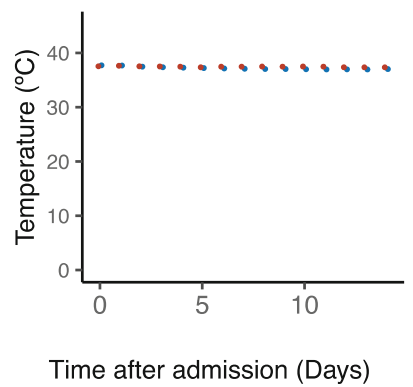

Heart rate

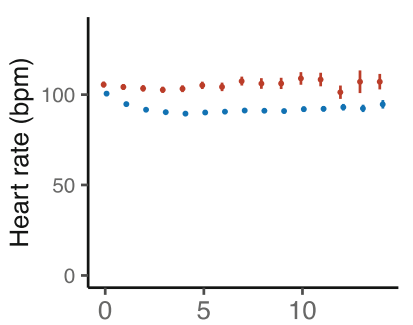

$\mathrm{SpO} 2$

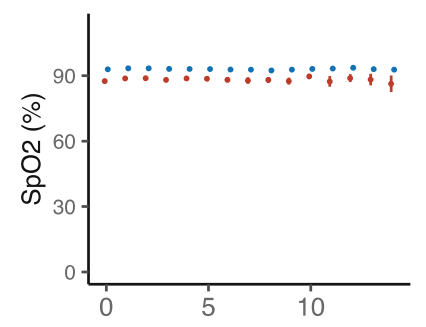

Figure 1 a Temporal change of vital signs in patients with COVID-19. BP, blood pressure; $\mathrm{SpO}_{2}$, peripheral oxygen saturation. b Temporal change of laboratory values in patients with COVID-19. WBC, white blood cell count; eGFR, estimated glomerular filtration rate; ALT, alanine aminotransferase; AST, aspartate aminotransferase; CRP, C-reactive protein; IL-6, interleukin-6; LDH, lactate dehydrogenase.

response to SARS-CoV infection ${ }^{17,}{ }^{18}$. Our findings are consistent with this theory, and we observed elevated IL- 6 as an independent prognostic risk factor, with higher levels in nonsurvivors. In hospitalized patients, we saw fluctuating IL-6 levels, with a significant increase seen on day 1 of admission and an increasing level trend that was more pronounced in non-survivors.

Thrombocytosis was associated with disease activity in SARS and was thought to be secondary to the direct effect of the virus or effect of inflammatory cytokines ${ }^{19}$. We observed a greater thrombocytosis during hospitalization in survivors than in non-survivors. A previous study of IL-6 in primates revealed that there is a dose-dependent response of thrombocytosis induced by IL- $6{ }^{20}$. The discrepancy between high IL-6 levels and lack of thrombocytosis in non-survivors could be explained by endothelial damage and subsequent platelet consumption from viral infection, impaired platelet release from megakaryocytes in the lung, or direct impairment of hematopoiesis ${ }^{21}$. This may suggest that the absence of reactive thrombocytosis may portend a poor response to SARS-CoV-2 infection.

Elevated D-dimer in COVID-19 patients has been described previously ${ }^{22,}{ }^{23}$. We report in this study its independent association with an increased risk of in-hospital mortality. Abnormal D-dimer alone is non-specific; however, the higher elevation in non-survivors suggests that coagulopathy, particularly disseminated intravascular coagulation (DIC), may contribute to mortality in COVID-19.
One of the functional receptors for pathogenic human coronavirus such as SARS-CoV is angiotensinconverting enzyme 2 (ACE2) ${ }^{24}$, and these receptors are expressed in heart tissues ${ }^{25}$. This suggests that SARSCoV-2 virus could directly affect the heart. Similar to the previous finding that showed an association of cardiac injury and a higher risk of in-hospital mortality ${ }^{26}$, we observed elevated troponin levels in hospitalized patients as a risk factor for increased mortality.

Hydroxychloroquine is an analog of chloroquine, a widely used anti-malarial with immunomodulatory effects ${ }^{27}$. In vitro studies have shown that hydroxychloroquine has activity against SARS-CoV-2 ${ }^{28}$. The clinical data of hydroxychloroquine in patients with COVID-19 come from small studies that have shown mixed results. Chen et al. randomized 30 hospitalized patients with COVID-19 to receive hydroxychloroquine $400 \mathrm{mg}$ daily for 5 days or placebo and found that $86.7 \%$ of the hydroxychloroquine group and $93.3 \%$ of the control group had negative throat swabs ${ }^{29}$. Chen et al. randomized 62 patients to hydroxychloroquine or placebo and reported shortened time to clinical recovery, fever resolution, and cough improvement in the hydroxychloroquine group ${ }^{30}$. Mahevas et al. reviewed 181 hospitalized patients with COVID-19 data who received hydroxychloroquine $600 \mathrm{mg}$ daily and reported no difference in outcomes, including in ICU admission and/or death at 7 days follow-up ${ }^{31}$. Another randomized trial of 150 hospitalized patients by Tang et al. did not show symptomatic improvement at 28 days or clearance of 
WBC

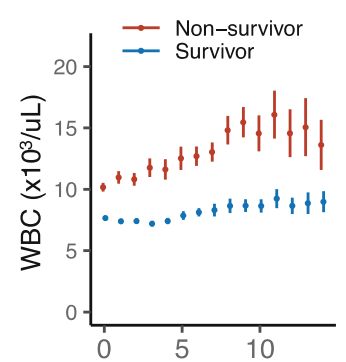

Platelet

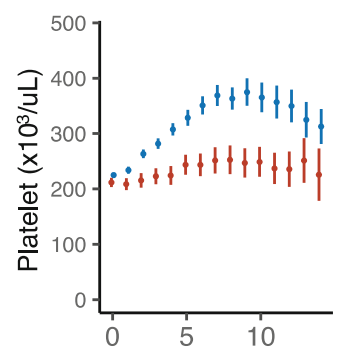

CRP

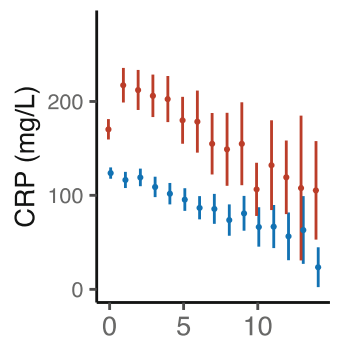

$\mathrm{LDH}$

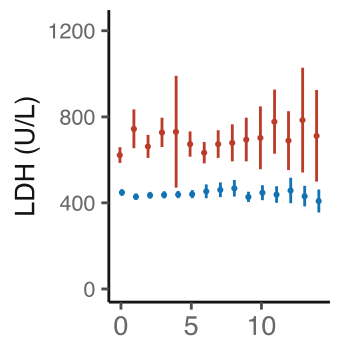

Neutrophil

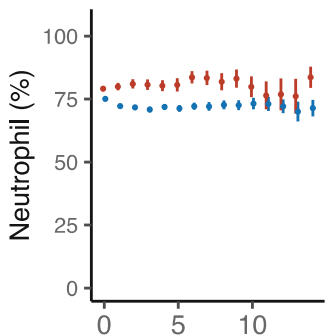

eGFR

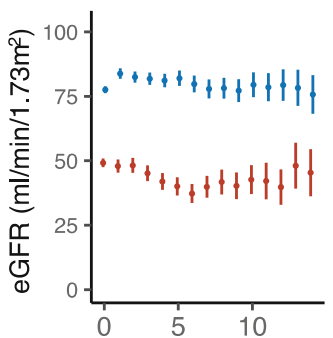

Procalcitonin

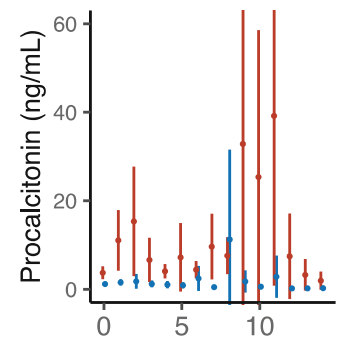

Fibrinogen

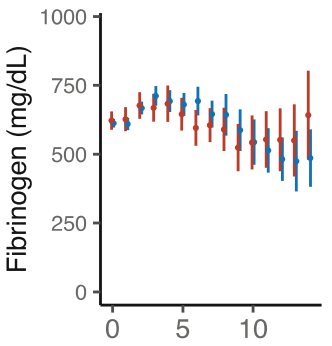

Lymphocyte

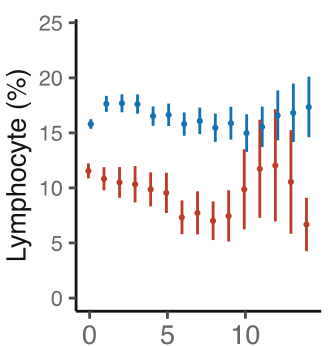

ALT

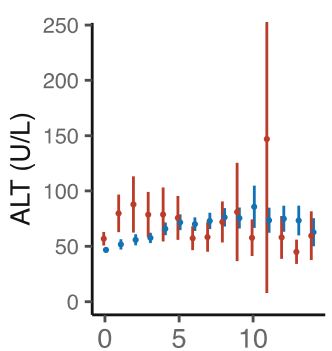

Ferritin

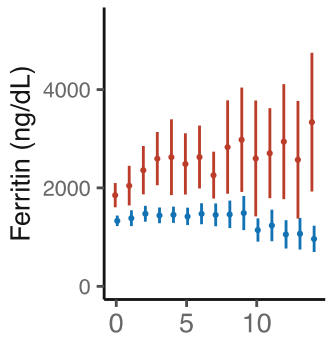

D-dimer

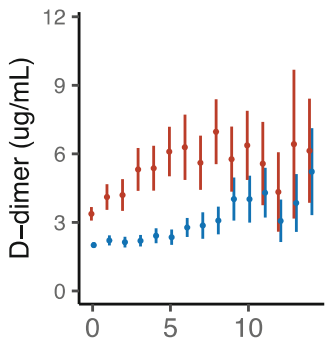

Hemoglobin

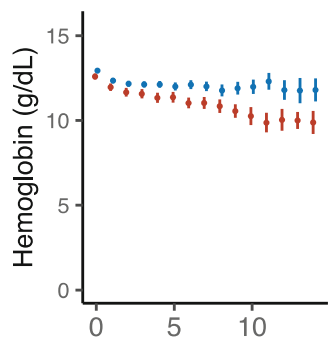

AST

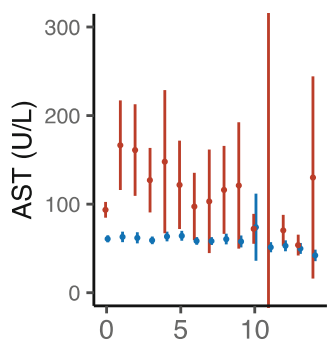

IL-6 (serum)

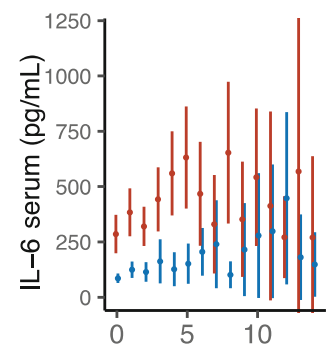

Troponin

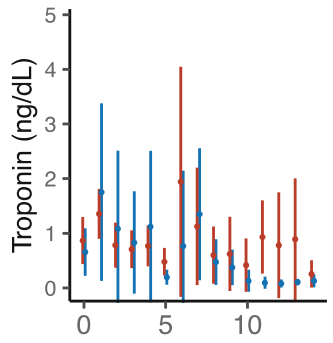

Time after admission (Days)

Fig. 1 (continued)

SARS-CoV-2 with hydroxychloroquine use ${ }^{32}$. We attempted to adjust for all known confounders between the groups who did and did not receive hydroxychloroquine using multivariate regression analyses and the IPTW method, which revealed that hydroxychloroquine use was associated with decreased risk of in-hospital mortality. Due to the inherent limitations of our retrospective study design, there was no conclusive determination on the efficacy of hydroxychloroquine in patients with COVID-19. More robust studies such as randomized clinical trials are needed.

Our study has several limitations. First, we have no longterm follow up data for ambulatory and discharged patients; hence, the clinical outcome observed may not be reflective of the true eventual outcome, particularly in the ambulatory group. Second, we have patients who remained hospitalized at the time of our analyses and did not have our outcomes, 
Table 3 Risk Factors Associated with In-Hospital Death

\begin{tabular}{|c|c|c|}
\hline & Hazard ratio $(95 \% \mathrm{CI})$ & $P$ value \\
\hline \multicolumn{3}{|l|}{ Age (reference: $<50$ years old) } \\
\hline $50-74$ years old & $2.34(1.47-3.71)$ & $<0.001$ \\
\hline$\geq 75$ years old & $4.85(2.75-8.56)$ & $<0.001$ \\
\hline Sex (Female) & $0.82(0.75-0.90)$ & $<0.001$ \\
\hline \multicolumn{3}{|l|}{ Race (reference: White) } \\
\hline African American & $0.78(0.65-0.95)$ & 0.011 \\
\hline Asian & $0.94(0.83-1.08)$ & 0.397 \\
\hline Others & $1.00(0.83-1.19)$ & 0.971 \\
\hline Cigarettes use history (reference: never smoker) & $1.01(0.90-1.13)$ & 0.916 \\
\hline Hypertension & $0.91(0.79-1.07)$ & 0.250 \\
\hline Diabetes & $0.92(0.73-1.16)$ & 0.481 \\
\hline Cancer & $1.08(0.84-1.40)$ & 0.550 \\
\hline Systolic blood pressure $<90 \mathrm{mmHg}$ & $1.38(1.06-1.80)$ & 0.017 \\
\hline \multicolumn{3}{|l|}{ Respiratory rate } \\
\hline $25-30$ per $\min$ & $1.43(1.13-1.83)$ & 0.004 \\
\hline$>30$ per $\min$ & $1.68(1.19-2.36)$ & 0.003 \\
\hline Peripheral oxygen saturation $\leq 92 \%$ & $2.12(1.56-2.88)$ & $<0.001$ \\
\hline Lymphocyte $\leq 12 \%$ & $1.12(0.97-1.29)$ & 0.110 \\
\hline \multicolumn{3}{|l|}{ Estimated glomerular filtration rate } \\
\hline $31-60 \mathrm{~mL} / \mathrm{min} / 1.73 \mathrm{~m}^{2}$ & $1.80(1.60-2.02)$ & $<0.001$ \\
\hline$<30 \mathrm{~mL} / \mathrm{min} / 1.73 \mathrm{~m}^{2}$ & $2.20(1.83-2.65)$ & $<0.001$ \\
\hline C-reactive protein $>150 \mathrm{mg} / \mathrm{L}$ & $1.03(0.78-1.36)$ & 0.815 \\
\hline Interleukin-6, serum $>100 \mathrm{pg} / \mathrm{mL}$ & $1.50(1.12-2.03)$ & 0.007 \\
\hline Lactate dehydrogenase $>440 \mathrm{U} / \mathrm{L}$ & $1.25(0.86-1.81)$ & 0.240 \\
\hline D-dimer $>2 \mu \mathrm{L} / \mathrm{mL}$ & $1.19(1.02-1.39)$ & 0.031 \\
\hline Troponin $>0.03 \mathrm{ng} / \mathrm{dL}$ & $1.41(1.23-1.62)$ & $<0.001$ \\
\hline Hydroxychloroquine use & $0.53(0.41-0.67)$ & $<0.001$ \\
\hline
\end{tabular}

such as discharge or mortality, and were excluded for our comparison of survivors and non-survivors. Third, due to limitations and local testing policy during the study duration, there are an unknown number of patients who were not diagnosed with COVID-19 because of a lack of severe symptoms and/or hospitalization. Fourth, we are not able to adjust for unknown confounders that may affect the true treatment effect. These limitations prevent any definitive conclusions on the efficacy of any treatment.

\section{CONCLUSIONS}

In this retrospective study of over 6000 ambulatory and hospitalized patients with COVID-19 in the New York City metropolitan area, age, male sex, tachypnea, low systolic blood pressure, low peripheral oxygen saturation, impaired renal function, elevated IL-6, elevated D-dimer, and elevated troponin were found to be risk factors for mortality. Hydroxychloroquine use was associated with decreased mortality.

\section{Acknowledgments:}

We thank the Mount Sinai Data Warehouse team for the COVID-19 database, and Norihiro Inoue $M D, P h D$, Hiroki Ueyama MD, Satoshi Miyashita MD, Misato Nagumo MD, and Mizuho Asada PhD for giving us critical comments and input.

Authors' Roles: TM and ES had the idea for and designed the study and had full access to all of the data in the study and take responsibility for the integrity of the data and the accuracy of the data analysis. TM and ES drafted the paper. TM, ES, HM, and TY did the analysis, and all authors critically revised the manuscript for important intellectual content and gave final approval for the version to be published. TM and ES collected the data. All authors agree to the accuracy or integrity of any part of the work are appropriately investigated and resolved.

Corresponding Author: Evan Siau, M.D.; Department of Medicine, Mount Sinai Beth Israel 281 First Ave, Box \#218, New York 10003, NY, USA (e-mail: Evan.Siau@mountsinai.org).

\section{Compliance with Ethical Standards:}

Informed consent was waived because of the de-identified and retrospective nature of the data. We confirm that we have read the Journal's position on issues involved in ethical publication and affirm that this work is consistent with those guidelines.

Conflict of Interest: The authors declare that they do not have a conflict of interest.

\section{REFERENCES}

1. Zhou $\mathbf{P}$, Yang $\mathbf{X L}$, Wang $\mathbf{X G}$, et al. A pneumonia outbreak associated with a new coronavirus of probable bat origin. Nature. 2020;579(7798):270-273.

2. Huang $\mathbf{C}$, Wang $\mathbf{Y}, \mathbf{L i} \mathbf{X}$, et al. Clinical features of patients infected with 2019 novel coronavirus in Wuhan, China. Lancet. 2020;395(10223):497506.

3. World Health Organization. Coronavirus Disease (COVID-19) Pandemic. https://www.who.int. Accessed 04/18, 2020.

4. New York City Department of Health and Mental Hygiene. COVID-19 Data. https://www1.nyc.gov/site/doh/covid/covid-19-data.page. Accessed 04/18, 2020.

5. Grasselli G, Zangrillo A, Zanella A, et al. Baseline Characteristics and Outcomes of 1591 Patients Infected With SARS-CoV-2 Admitted to ICUs of the Lombardy Region, Italy. JAMA. 2020.

6. Guan WJ, Ni ZY, Hu Y, et al. Clinical Characteristics of Coronavirus Disease 2019 in China. N Engl J Med. 2020.

7. Chen $\mathbf{N}$, Zhou M, Dong $\mathbf{X}$, et al. Epidemiological and clinical characteristics of 99 cases of 2019 novel coronavirus pneumonia in Wuhan, China: a descriptive study. Lancet. 2020;395(10223):507-513.

8. Chen T, Wu D, Chen H, et al. Clinical characteristics of 113 deceased patients with coronavirus disease 2019: retrospective study. BMJ. 2020;368:m1091. 
9. Wang D, Hu B, Hu C, et al. Clinical Characteristics of 138 Hospitalized Patients With 2019 Novel Coronavirus-Infected Pneumonia in Wuhan, China. JAMA. 2020.

10. Xu XW, Wu XX, Jiang XG, et al. Clinical findings in a group of patients infected with the 2019 novel coronavirus (SARS-Cov-2) outside of Wuhan, China: retrospective case series. BMJ. 2020;368:m606.

11. Zhou F, Yu T, Du R, et al. Clinical course and risk factors for mortality of adult inpatients with COVID-19 in Wuhan, China: a retrospective cohort study. Lancet. 2020;395(10229): 1054-1062.

12. Wu Z, McGoogan JM. Characteristics of and Important Lessons From the Coronavirus Disease 2019 (COVID-19) Outbreak in China: Summary of a Report of 72314 Cases From the Chinese Center for Disease Control and Prevention. JAMA. 2020.

13. World Health Organization. Middle East respiratory syndrome coronavirus (MERS-CoV). https://www.who.int/emergencies/mers-cov/en. Accessed 04/18, 2020.

14. World Health Organization. Cumulative Number of Reported Probable Cases of Severe Acute Respiratory Syndrome (SARS). https://www.who. int/csr/sars/country/en/. Accessed 04/18, 2020.

15. Perlman S, Netland J. Coronaviruses post-SARS: update on replication and pathogenesis. Nat Rev Microbiol. 2009;7(6):439-450.

16. Henry BM, Lippi G. Chronic kidney disease is associated with severe coronavirus disease 2019 (COVID-19) infection. Int Urol Nephrol. 2020.

17. Thiel V, Weber F. Interferon and cytokine responses to SARS-coronavirus infection. Cytokine Growth Factor Rev. 2008;19(2):121-132.

18. Perlman S, Dandekar AA. Immunopathogenesis of coronavirus infections: implications for SARS. Nat Rev Immunol. 2005;5(12):917-927.

19. Wong RS, Wu A, To KF, et al. Haematological manifestations in patients with severe acute respiratory syndrome: retrospective analysis. BMJ. 2003;326(7403): 1358-1362.

20. Asano S, Okano A, Ozawa $\mathbf{K}$, et al. In vivo effects of recombinant human interleukin-6 in primates: stimulated production of platelets. 1990

21. Yang $\mathbf{M}, \mathbf{N g} \mathbf{M H}, \mathbf{L i} \mathbf{C K}$. Thrombocytopenia in patients with severe acute respiratory syndrome (review). Hematology. 2005;10(2):101-105.

22. Han H, Yang $\mathbf{L}$, Liu R, et al. Prominent changes in blood coagulation of patients with SARS-CoV-2 infection. Clin Chem Lab Med. 2020.
23. Lippi G, Favaloro EJ. D-dimer is Associated with Severity of Coronavirus Disease 2019: A Pooled Analysis. Thromb Haemost. 2020.

24. Li W, Moore MJ, Vasilieva N, et al. Angiotensin-converting enzyme 2 is a functional receptor for the SARS coronavirus. Nature. 2003;426(6965):450-454.

25. Gallagher PE, Ferrario CM, Tallant EA. Regulation of ACE2 in cardiac myocytes and fibroblasts. Am J Physiol Heart Circ Physiol. 2008;295(6):H2373-2379.

26. Shi S, Qin M, Shen B, et al. Association of Cardiac Injury With Mortality in Hospitalized Patients With COVID-19 in Wuhan, China. JAMA Cardiol. 2020.

27. Keyaerts E, Vijgen L, Maes P, Neyts J, Van Ranst M. In vitro inhibition of severe acute respiratory syndrome coronavirus by chloroquine. Biochem Biophys Res Commun. 2004;323(1):264-268.

28. Yao X, Ye F, Zhang M, et al. In Vitro Antiviral Activity and Projection of Optimized Dosing Design of Hydroxychloroquine for the Treatment of Severe Acute Respiratory Syndrome Coronavirus 2 (SARS-CoV-2). Clin Infect Dis. 2020.

29. Chen J, Liu L, Liu P, Xu Q , Xia L, Ling Y, Huang D, Song S, Zhang D, Gian Z, Li T, Shen Y, Lu H. A pilot study of hydroxychloroquine in treatment of patients with common coronavirus disease-19 (COVID-19). $J$ Zhejiang Univ (Med Sci). 2020;49(1):0-0.

30. Chen Z, Hu J, Zhang Z, et al. Efficacy of hydroxychloroquine in patients with COVID-19: results of a randomized clinical trial. medRxiv. 2020:2020.2003.2022.20040758.

31. Mahevas M, Tran V-T, Roumier M, et al. No evidence of clinical efficacy of hydroxychloroquine in patients hospitalized for COVID-19 infection with oxygen requirement: results of a study using routinely collected data to emulate a target trial. medRxiv. 2020:2020.2004.2010.20060699.

32. Tang W, Cao Z, Han $\mathbf{M}$, et al. Hydroxychloroquine in patients with COVID-19: an open-label, randomized, controlled trial. medRxiv. 2020:2020.2004.2010.20060558.

Publisher's Note: Springer Nature remains neutral with regard to jurisdictional claims in published maps and institutional affiliations. 